\title{
Die EU und der Kampf gegen den Terrorismus - die schwierige Balance zwischen Sicherheit und Freiheit
}

\author{
Martin Kahl*
}

\begin{abstract}
The terrorist attacks in Madrid (March 2004) and London (July 2005) have increased the pressure on the EU to improve strategies and instruments of its counter-terrorism policy. The specific structure of islamic terrorism calls for adjustments with regard to new terrorist actors, motives and potentials. The design and development of an effective policy against terrorism, however, faces a wide range of challenges. One of the most fundamental is the problem of finding an appropriate balance between internal security and the protection of civil liberties of EU citizens. In dealing with terrorism the EU has already decided for several measures that not only undermine civil liberties but are of questionable effectiveness.
\end{abstract}

Keywords: Europäische Union, islamistischer Terrorismus, Terrorismusbekämpfung, Bürgerrechte

\section{Nach den Anschlägen von Madrid und London: Probleme der EU-Terrorismuspolitik}

$\mathrm{D}$ ie Anschläge von Madrid (März 2004) und London (Juli 2005) haben den Handlungsdruck auf die Europäische Union (EU) und ihre Mitgliedstaaten, wirksame Strategien und Instrumente vor allem zur Bekämpfung des islamistischen Terrorismus zu entwickeln und umzusetzen, noch einmal deutlich erhöht. Die Entwicklung einer effektiven Antiterrorismuspolitik durch die EU ist jedoch mit einer Reihe von Problemen belastet.

Da die Aufgabe der Terrorismusbekämpfung in erster Linie den Mitgliedstaaten zufällt, hat die EU eigene Handlungskompetenzen bisher nur in geringem Umfang aufbauen können. Die Union verfügt noch immer nicht über eigenständige Kompetenzen bei der Strafverfolgung, der nachrichtendienstlichen Informationsbeschaffung und im Bereich operativer Maßnahmen. Selbst diejenigen europäischen Institutionen, die im Zuge der Terrorismusbekämpfung neu geschaffen oder personell aufgestockt worden sind, wie etwa der neu eingesetzte EUKoordinator für die Terrorismusbekämpfung, besitzen wenig eigenständige Befugnisse. Das Spannungsverhältnis zwischen nationalstaatlichen und gemeinschaftlichen Sicherheitskonzepten hat zudem immer wieder zu erheblichen Abstimmungsproblemen zwischen den Mitgliedstaaten und EU-Organen bzw. -Behörden wie Europol (Europäisches Polizeiamt) und Eurojust (Europäische Staatsanwaltschaftsbehörde) geführt.

Bereits nach den Anschlägen vom 11. September 2001 waren auf EU-Ebene überdies zwar Verbesserungen im Bereich der Kooperation von Polizei und Justiz, des Informationsaustausches, bei den Grenzkontrollen, der Abwicklung von Visaerteilungen und Einreisegenehmigungen, der Flugsicherheit und bei Maßnahmen zur Bekämpfung der Finanzierung des Terrorismus beschlossen worden. Die EU hatte zudem begonnen, neue Institutionen, Kooperationsformen und -foren, strafprozessrechtliche Verfahren und Informationssysteme zu schaffen sowie einen breiten Katalog aus Einzelmaßnahmen zur Terrorismusbekämpfung zu entwickeln. Viele dieser Maß-

\footnotetext{
* Dr. Martin Kahl, Wissenschaftlicher Mitarbeiter am Zentrum für Europäische Friedens- und Sicherheitsstudien (ZEUS) am Institut für Friedensforschung und Sicherheitspolitik an der Universität Hamburg (IFSH).
}

nahmen sind von den EU-Mitgliedstaaten jedoch nur schleppend umgesetzt worden.

Darüber hinaus bedingt die spezifische Problemstruktur des islamistischen Terrorismus, dass die Terrorismusbekämpfungspolitik der EU sich auf neue Akteure, Motive und Potenziale einstellen muss. Da es bei dem in Europa virulenten islamistischen Terrorismus mehr Kontingenz und Singularitäten gibt als Regelmäßigkeiten, steht die Politik vor großen Herausforderungen, nicht zuletzt angesichts der Aufgabe, einerseits die Handlungsfreiheit der Terroristen einzuschränken und andererseits bürgerliche Freiheitsrechte zu wahren. Unter dem nach den Anschlägen von Madrid und London entstandenen Handlungsdruck sind von den Mitgliedstaaten und der EU jedoch Maßnahmen beschlossen und teilweise bereits umgesetzt worden, die nicht nur mit Blick auf ihre Wirksamkeit als problematisch gelten müssen, sondern auch die Balance zuungunsten liberaler Werte verschoben haben.

\section{Der Maßnahmenkatalog der EU zur Terroris- musbekämpfung}

Die EU schließt ein militärisches Vorgehen gegen Terroristen und sie unterstützende Staaten grundsätzlich nicht aus, die von ihr ergriffenen Maßnahmen zeigen jedoch, dass sie Terrorismus als spezifische Form von Kriminalität betrachtet, auf die primär kriminalpräventiv und mit Mitteln der Strafverfolgung durch Polizei und Justiz zu antworten ist. Bereits vor dem 11. September 2001 hat die EU bei der Bekämpfung von Gefahren für ihre innere Sicherheit deutliche Akzente auf Risiken durch »soft security issues« (organisiertes Verbrechen, Korruption, Menschenhandel, illegale Einwanderung, illegaler Handel mit Waffen und Drogen, Terrorismus) gelegt, die oftmals auch eine grenzüberschreitende Dimension haben und in vielfacher Weise miteinander zusammenhängen. ${ }^{1}$ So ist es kein Zufall, dass viele Maßnahmen, die von den Mitgliedstaaten der

1 Siehe hierzu Barry Buzan, Societal Security: The Concept, in: OleWaever/ Barry Buzan/Morten Kelstrup/Pierre Lemaitre, Identity, Migration and the New Security Agenda in Europe, New York 1992, S. 17-40; Alessandro Politi, European Security: The New Transnational Risks, Chaillot Paper 29, WEU Institute for Security Studies, Paris 1997; Bernhard Zangl/Michael Zürn, Frieden und Krieg. Sicherheit in der nationalen und postnationalen Konstellation, Frankfurt am Main 2003. 
EU in ihrem ersten Aktionsplan nach dem 11. September 2001 als Instrumente zur Terrorbekämpfung beschlossen wurden, bereits zuvor im Zuge der Bekämpfung grenzüberschreitender Kriminalität verhandelt oder eingeführt worden waren. ${ }^{2}$ Der revidierte Aktionsplan zur Bekämpfung des Terrorismus vom Juni 2005 listet mehr als 180 verschiedene Einzelmaßnahmen und Aktionen auf - wobei deutliches Gewicht auf die polizeiliche und justizielle Zusammenarbeit in Strafsachen (die so genannte »dritte Säule « der EU) gelegt wird. ${ }^{3}$ In dem Plan sind jedoch auch Maßnahmen aus der »ersten Säule« (Asyl, Einwanderung) und der »zweiten Säule« (Gemeinsame Außenund Sicherheitspolitik) enthalten. Dies trägt der Auffassung von Rat und Kommission Rechnung, dass binnenorientierte Antiterrormaßnahmen, die vor allem die dritte Säule betreffen, zur Entfaltung ihrer Wirksamkeit flankierender Maßnahmen aus anderen Tätigkeitsbereichen der Union bedürfen. Im November 2005 schließlich hat der Rat eine umfassende Strategie zur Terrorismusbekämpfung verabschiedet, die Prävention, Schutz, Verfolgung und Reaktion auf Terrorangriffe durch außen- und binnengerichtete Maßnahmen zusammenbindet. ${ }^{4}$ In der EU hat sich also die Auffassung durchgesetzt, dass zur Verhütung des Terrorismus die Zusammenarbeit mit Drittstaaten, die Sicherung der Außengrenzen und Maßnahmen im Bereich der inneren Sicherheit als unteilbares Ganzes betrachtet werden müssen.

\subsection{Zusammenarbeit mit Drittstaaten bei der Terrorismusbekämpfung}

Der Regelungsbedarf in Bezug auf Drittstaaten wird von der EU als so umfassend erachtet, dass über rein »defensive« Abschottungsmaßnahmen hinausgegangen und diese Staaten in die Lage versetzt werden sollen, sicherheitsrelevante Aufgaben für die EU wahrzunehmen. Dahinter steht die Erkenntnis, dass eigene Grenzkontrollen in einem global vernetzten Staatenund Gesellschaftssystem wie der EU, in dem innen und außen durch vielgestaltige grenzüberschreitende Transaktionen (Personen, Waren, Kommunikations- und Finanzströme) verschwimmen, nicht ausreichen. ${ }^{5}$ Wenn die Außengrenzen der EU so verwaltet werden sollen, dass auf der einen Seite Personen ohne größere Behinderungen reisen können, illegale Einwanderung und die Einschleusung von Terroristen auf der anderen verhindert wird, ist dies nach Ansicht des Rates der EU eine Angelegenheit herkömmlicher Grenzkontrolle,

2 Europäischer Rat, Schlussfolgerungen und Aktionsplan anlässlich der außerordentlichen Tagung des Europäischen Rates am 21. September 2001 in Brüssel, SN 140/01.

3 Vgl. Council of the European Union, EU Plan of Action on Combating Terrorism - Update, Brussels, 10 June 2005, 9809/1/05, REV 1 ADD 1.

4 Rat der Europäischen Union, Strategie der Europäischen Union zur Terrorismusbekämpfung, Brüssel, 30.11.2005, 14469/4/05 REV 4, S. 6.

5 Edward S. Cohen, Globalization and the Boundaries of the State: A Framework for Analyzing the Changing Practice of Sovereignty, in: Governance 1/2001, S. 75-97; Peter Andreas, Redrawing the Line. Borders and Security in the Twenty-First Century, in: International Security 2/2003, S. 78-111; Malcolm Anderson/Joanna Apap (Hrsg.), Police and Justice Co-operation and the New European Borders, The Hague/London/New York 2002; Malcolm Anderson/Eberhard Bort, The Frontiers of the European Union, Basingstoke u.a. 2001; Eberhard Bort, Illegal Migration and Cross-border Crime, in: Jan Zielonka (Hrsg.), Europe Unbound - Enlarging and reshaping the boundaries of the European Union, London/New York 2002, S. 190-212; Albrecht Funk, Policing Europe: Border Controls and European Integration, in: Stefan Immerfall (Hrsg.), Territoriality in the Globalizing Society, Berlin/New York 1998, S. 91-109. der Sicherheit von Reisedokumenten, der Visaerteilung oder der Erhebung und des Austausches von Daten über Reisende, ${ }^{6}$ zusätzlich aber auch einer langen Reihe von Maßnahmen, die sich nicht nur auf den Binnenbereich der EU beziehen, sondern auch auf Drittstaaten. ${ }^{7}$ Gegenüber Drittstaaten geht es der EU vor allem um den Aufbau oder die Verbesserung von Kapazitäten der Sicherheitsapparate in den Zielstaaten sowie um die Übertragung eines weit gefassten Aufgabenkatalogs, den diese Staaten erfüllen sollen. Hierzu gehören unter anderem die Übernahme internationaler Abkommen zur Terrorismusbekämpfung, ferner der Austausch terrorismusrelevanter Informationen, Erfahrungen und Techniken, Maßnahmen zur Unterbindung der Finanzierung des Terrorismus, sowie aufgrund seiner angenommenen funktionalen Nähe zum Terrorismus vor allem auch der Grenzschutz. ${ }^{8}$

Zur Durchsetzung dieser Politik dient der EU eine weite Reihe von Instrumenten, darunter die Aufnahme von »Terrorismusklauseln « in bilaterale Abkommen, ${ }^{9}$ Aktionspläne im Rahmen der Europäischen Nachbarschaftspolitik (ENP), ${ }^{10}$ die operative Zusammenarbeit mit Europol, Eurojust, der Europäischen Polizeiakademie und der Agentur für Außengrenzen sowie die Außenhilfepolitik. ${ }^{11}$ Dabei reichen die Mechanismen, die die EU einsetzt, von technischer Unterstützung bis hin zum Hebel der Konditionalität, durch die eine Intensivierung der Handelsbeziehungen an bestimmte operativ-administrative Forderungen geknüpft wird. In gezielte Maßnahmen zur Verbesserung der Terrorismusbekämpfung sollen insbesondere Marokko und Algerien, aber auch ausgesprochene Problemstaaten wie Libyen einbezogen werden. ${ }^{12}$ Der EU ist in diesem Zusammenhang vorgehalten worden, sie versuche durch solche »vorgelagerten Strategien « vor allem die restriktiven Bestandteile ihrer Einwanderungspolitik in die Nachbarstaaten zu exportieren ${ }^{13}$ und verliere über die Stärkung der Sicherheitsapparate (semi)autoritärer Staaten sowie die generelle

6 Council of the European Union, Presidency Programme on Asylum and Immigration, Brussels, 29 June 2005, 10703/05; Mitteilung der Kommission an den Rat und das Europäische Parlament, Auf dem Weg zu einem integrierten Grenzschutz an den Außengrenzen der EU-Mitgliedstaaten, KOM(2002) 233 endgültig.

7 Vorschlag für eine Entscheidung des Europäischen Parlaments und des Rates zur Einrichtung des Außengrenzenfonds für den Zeitraum 2007-2013 innerhalb des generellen Programms »Solidarität und Steuerung der Migrationsströme« \{SEK(2005) 435\} KOM(2005) 123 endgültig, COD 2005/0047.

8 Auch das »Haager Programm « der EU sieht in diesem Sinne eine engere Zusammenarbeit mit Drittstaaten bei grenzüberschreitenden Problemen vor. Ziel des Programms ist unter anderem die Verbesserung der Regulierung von Wanderungsbewegungen und die Kontrolle der Außengrenzen der Union, des Kampfes gegen organisierte grenzüberschreitende Kriminalität und der Bekämpfung der Bedrohung durch den Terrorismus. Haager Programm zur Stärkung von Freiheit, Sicherheit und Recht in der Europäischen Union, Abl. 2005/C 53/01 (3.3.2005).

9 Vgl. Haager Programm zur Stärkung von Freiheit, Sicherheit und Recht in der Europäischen Union, Abl. 2005/C 53/01 (3.3.2005), S. 1-14.

10 Vgl. Kommission der Europäischen Gemeinschaften, Mitteilung der Kommission: Europäische Nachbarschaftspolitik - Strategiepapier, Brüssel, 12.5.2004, KOM(2004) 373 endgültig, S. 6.

11 Vgl. Kommission der Europäischen Gemeinschaften, Mitteilung der Kommission. Eine Strategie für die Außendimension des Raums der Freiheit, der Sicherheit und des Rechts, Brüssel, 12.10.2005, KOM(2005) 491 endgültig, S. 3-10.

$12 \mathrm{Vgl}$. Rat der Europäischen Union: Durchführung des Aktionsplans zur Bekämpfung des Terrorismus, Brüssel, 29.11.2005, 14734/1/05 REV 1, S. 12; Rat der Europäischen Union: 14469/4/05 REV 4, S. 7.

13 Elspeth Guild, Moving the Borders of Europe. Inaugural Lecture on the Occasion of the Assumption of the Professorship at the University of Nijmegen, 2001, http://www. jur.kun.nl/cmr/ articles/oratieEG.pdf; Christina Boswell, The »External Dimension « of EU Immigration and Asylum Policy, in: International Affairs 3/2003, S. 619-638; Gil Loescher/James Milner, The Missing Link: The Need for Comprehensive Engagement in Regions of Refugee Origin, in: International Affairs 3/2003, S. 595-617. 
Stabilisierung der Regime dieser Staaten das Ziel der Demokratisierung und die Durchsetzung menschenrechtlicher Belange aus den Augen.

\subsection{Terrorismusabwehr an den Grenzen und im Binnenraum der EU}

Ein effektiver Schutz des Raums der Freiheit der Sicherheit und des Rechts bedeutet aus Sicht der EU nicht nur, Terroristen und ihre Helfer daran zu hindern, auf das Territorium der EU zu gelangen, sondern auch solche, die sich bereits innerhalb der EU befinden, rechtzeitig ausfindig machen zu können. Dabei legt sie deutliches Gewicht auf die Identifizierung von Drittstaatsangehörigen, insbesondere solchen, die sich unter Verwendung gefälschter Papiere in der EU aufhalten. Terroristen sollen vor allem durch die Intensivierung einer Vielzahl von Kontrollen, eine bessere Vernetzung nationaler und europäischer Sicherheitsbehörden (Polizei, Justiz, Einwanderungsbehörden und Nachrichtendienste) sowie durch den Austausch polizeilicher und justizieller Erkenntnisse insbesondere über Europol und Eurojust frühzeitig aufgespürt und ihnen ihre finanziellen, ökonomischen und operativen Ressourcen entzogen werden.

Einem Vorschlag der EU-Kommission vom November 2005 zufolge sollen die Sicherheitsbehörden der Mitgliedstaaten sowie Europol Zugang zum neu eingerichteten Visa-Informationssystem (VIS) erhalten und Datenabfragen im Zusammenhang mit terroristischen Straftaten vornehmen können. ${ }^{14}$ Auch für das Schengener Informationssystem der zweiten Generation (SIS II) hat sie einen erweiterten Informationszugang für diese Behörden angeregt. Zudem hat sie sich für eine Weiterentwicklung und Vernetzung der drei für den Bereich Justiz und Inneres relevanten europäischen Datenbanken - neben dem VIS und dem SIS die Datenbank der Fingerabdrücke von Asylbewerbern und illegalen Einwanderern (EURODAC) - ausgesprochen. ${ }^{15}$

Im Zuge der binnengerichteten Terrorismusbekämpfung werden jedoch nicht nur Informationssysteme ausgebaut, die Daten von Drittstaatsangehörigen enthalten - auch wenn hier das größte Gewicht liegt - sondern auch solche, in denen strafrechtlich relevante Daten von EU-Bürger(-inne)n gespeichert sind. SIS II, dessen Entwicklung nach dem 11. September 2001 intensiv vorangetrieben worden ist, soll die Befugnisse und Leistungsmerkmale gegenüber dem bestehenden System erheblich erweitern. ${ }^{16}$ Über die endgültige Ausgestaltung des

14 Vgl. Kommission der Europäischen Gemeinschaften, Vorschlag für einen Beschluss des Rates über den Zugang der für die innere Sicherheit zuständigen Behörden der Mitgliedstaaten und von Europol zum Visa-Informationssystem (VIS) für Datenabfragen zum Zwecke der Prävention, Aufdeckung und Untersuchung terroristischer und sonstiger schwerwiegender Straftaten, Brüssel, 24.11.2005, KOM(2005) 600 endgültig, S. 2-6.

15 Vgl. Kommission der Europäischen Gemeinschaften, Mitteilung der Kommission an den Rat und das Europäische Parlament über die Verbesserung der Effizienz der europäischen Datenbanken im Bereich Justiz und Inneres und die Steigerung ihrer Interoperabilität sowie der Synergien zwischen ihnen, Brüssel, 24.11.2005, KOM(2005) 597 endgültig, S. 6-12.

16 Vgl. Statewatch Analysis, SIS II: fait accompli? Construction of EU's Big Brother database underway, May 2005, http://www.statewatch.org/news/ index.html. Das Schengen Informationssystem der ersten Generation umfasst bereits Kategorien wie die Aufenthaltsermittlung von Zeugen und Beschuldigten, die verdeckte Registrierung von verdächtigen Personen sowie die Sachfahndung nach Kraftfahrzeugen oder Identitätspapieren.
Systems wird noch verhandelt, es kann jedoch davon ausgegangen werden, dass insbesondere der Zugang zu Daten über EU-Bürger erweitert sowie neue Ausschreibungs- und Datenkategorien hinzugefügt und neue Verknüpfungsmöglichkeiten geschaffen werden.

Vor allem Deutschland und Spanien haben für SIS II weitreichende Vorschläge eingebracht. Sie haben die Aufnahme zusätzlicher biografischer und polizeilicher Personenmerkmale gefordert, sowie die Schaffung automatisierter Verknüpfungsmöglichkeiten zwischen verschiedenen Ausschreibungskategorien, die Verlängerung von Ausschreibungs- und Datenlöschungsfristen, die Einführung einer speziellen Terroristendatenbank und die Schaffung weiterer Speicher-, Übermittlungs- und Abrufmöglichkeiten für biometrische Daten, etwa digitalisierte Gesichtsbilder oder genetische Fingerabdrücke. Neben Visa-, Pass- und Zollbehörden, Polizei, nationalen Justizbehörden sowie zu Teilzugriffen berechtigten Behörden (Europol, Eurojust, Geheimdienste) sollen in Zukunft zusätzlich Kfz-Behörden, aber auch nichtstaatliche Stellen wie Schutzeinrichtungen der Kreditwirtschaft, Rechtsanwälte, Notare oder Fluggesellschaften sowie im Rahmen der Terrorismusbekämpfung bestimmte Stellen von Nicht-EU-Staaten auf SIS II zugreifen können.

Wird auch nur ein Teil dieser Vorschläge zur Aufnahme neuer Datenkategorien und zur Bereitstellung für neue Nutzer umgesetzt, dann wird das SIS von einer polizeilichen Ausschreibungsdatei zu einem umfassenden polizeilichen Informationsund Analysesystem, das eine erhebliche Anzahl von Daten über nahezu alle EU-Bürger(-innen) enthält. Aufgrund seiner komplexen Struktur wäre es zudem von den nationalen und supranationalen Aufsichts- und Datenschutzeinrichtungen nur noch schwer zu kontrollieren.

Wird durch solche Maßnahmen auf der einen Seite eine Ausweitung und Intensivierung der Erfassung von Daten von Drittstaatsangehörigen und EU-Bürger(-inne)n projektiert, so bestehen paradoxerweise gerade beim Informationsaustausch strafrechtlich relevanter Daten und geheimdienstlicher Erkenntnisse zwischen den Mitgliedstaaten noch immer erhebliche Probleme. ${ }^{17}$ Um hier Abhilfe zu schaffen, hat der Rat im Sommer 2005 zwei Rechtsakte angenommen, die auch für die Terrorismusbekämpfung relevant sind: Als Übergangsmaßnahme wurde zum einen ein Beschluss über den Austausch von Informationen aus den Strafregistern angenommen, ${ }^{18}$ zum anderen wurden die Mitgliedstaaten verpflichtet, einander sowie Europol und Eurojust Daten über laufende strafrechtliche Ermittlungen und Verfolgungen im Bereich des Terrorismus zur Verfügung zu stellen. ${ }^{19} \mathrm{Um}$ im Bereich des Austauschs von strafrechtlich relevanten Daten von einer Ad-hoc-Zusammenarbeit zu systematischer polizeilicher Zusammenarbeit zu gelangen, soll zudem dem Grundsatz der Verfügbarkeit von Strafverfolgungsinformationen Geltung

17 Der Rat der EU hat dies selbst deutlich bemängelt. Vgl. Rat der Europäischen Union: 14734/1/05 REV 1, a.a.O., S. 3.

18 Vgl. Council of the European Union, Proposal for a Council Decision on the Exchange of Information Extracted from Criminal Records, Brussels, 28 July 2005, 11569/05, S. $1-4$

19 Beschluss 2005/671/JI des Rates vom 20. September 2005 über den Informationsaustausch und die Zusammenarbeit betreffend terroristische Straftaten, Abl. L 253 vom 29.9.2005, S. 22-24. 
verschafft werden. Dies bedeutet, dass Strafverfolgungsbeamte Informationen aus anderen Mitgliedstaaten zu den gleichen Bedingungen erhalten sollen, die auch für innerstaatliche Auskunftsersuchen gelten. ${ }^{20}$

Nachdem sich aus Sicht des Rates der Europäische Haftbefehl als wichtiges Werkzeug für die Verfolgung von Terroristen über die Binnengrenzen der EU hinweg erwiesen hat, sollen darüber hinaus weitere Maßnahmen zur Verbesserung der Zusammenarbeit in strafrechtlichen Angelegenheiten angegangen werden, so etwa die gegenseitige Anerkennung gerichtlicher Entscheidungen und die Europäische Beweisanordnung, durch die Mitgliedstaaten in die Lage versetzt werden sollen, Beweise aus anderen EU-Ländern zu erhalten. ${ }^{21} \mathrm{Nach}$ Auffassung des Rates soll es hierdurch einfacher werden, auch Terroristen zu überführen. ${ }^{22}$ Auch mit diesen Maßnahmen im Bereich der Erhebung und des Austauschs von strafrechtlich relevanten Informationen sind aufgrund materiell- und verfahrensrechtlicher Unterschiede zwischen den EU-Staaten erhebliche Probleme aus der Perspektive des Grundrechts- und des Datenschutzes verbunden.

\section{Terrorismusbekämpfung und der Schutz der Freiheitsrechte}

Auch wenn die EU im Vergleich zu ihren Mitgliedstaaten noch über wenig eigene Kompetenzen bei der Terrorismusbekämpfung verfügt und ihre Politik in diesem Bereich durch vielerlei Ineffizienzen gekennzeichnet ist, so bedrohen viele der bereits umgesetzten und vor allem die geplanten präventiven Überwachungsmaßnahmen sowie die oben beschriebenen Ausweitungen der Befugnisse von Strafverfolgungsbehörden und Geheimdiensten in erheblichem Maße Freiheits- und Bürgerrechte. Die EU hat in dieser Hinsicht bereits eine Reihe bedenklicher Maßnahmen eingeleitet, die tief in die Selbstbestimmungs- und Persönlichkeitsrechte der Bürger und Bürgerinnen eingreifen, darunter die Übermittlung persönlicher Daten von EU-Bürger(-inne)n, die Flugreisen in die USA unternehmen, an amerikanische Sicherheitsbehörden ${ }^{23}$ und die verdachtsunabhängige, mindestens sechsmonatige Speicherung

20 Vgl. Kommission der Europäischen Gemeinschaften, Vorschlag für einen Beschluss des Rates über die Übermittlung von aus den Tätigkeiten der Sicherheits- und Nachrichtendienste resultierenden Informationen über terroristische Straftaten, Brüssel, 22.12.2005 KOM(2005) 695 endgültig, S. 10-11.

21 Hierüber hat der Rat im Juni 2006 grundsätzlich Einigung erzielt.

22 Vgl. Rat der Europäischen Union: 14734/1/05 REV 1, a.a.O., S. 5.

23 Beschluss 2004/496/EG des Rates vom 17. Mai 2004 über den Abschluss eines Abkommens zwischen der Europäischen Gemeinschaft und den Vereinigten Staaten von Amerika über die Verarbeitung von Fluggastdatensätzen und deren Übermittlung durch die Fluggesellschaften an das Bureau of Customs and Border Protection des United States Department of Homeland Security (ABl. L 183, vom 20.5.2004, S. 83, berichtigt im ABl.2005, L 255 vom 30.9.2005, S. 168); Kommission der Europäischen Gemeinschaften, Entscheidung 2004/535/EG der Kommission vom 14. Mai 2004 über die Angemessenheit des Schutzes der personenbezogenen Daten, die in den Passenger Name Records enthalten sind, welche dem United States Bureau of Customs and Border Protection übermittelt werden, ABl. L 235 vom 6.7.2004, S. 11 Der Europäische Gerichtshof hat den Beschluss des Rates und die Entscheidung der Kommission durch ein Urteil am 30. Mai 2006 für nichtig erklärt (http://curia.eu.int/jurisp/cgi-bin/ form.pl?lang=DE\& Submit=rechercher \&numaff=C-317/04). der Telefon- und Internetdaten jedes EU-Bürgers. ${ }^{24}$ Beispiele für Kontroll- bzw. Überwachungsmaßnahmen, die sämtliche EU-Bürger, unabhängig von Straftatbeständen betreffen, ist außerdem das Programm zur Fälschungssicherheit von Reisedokumenten, in dessen Rahmen bis 2008 zwei biometrische Merkmale (Gesichtserkennung und Fingerabdruck) in Pässe aufgenommen werden sollen. Ferner ist ein Informationssystem projektiert, mit dessen Hilfe Angaben über verloren gegangene Pässe unter Rückgriff auf das SIS und die Interpol-Datenbank ausgetauscht werden können. Im letzten Jahresbericht zur Tätigkeit der »Artikel 29«-Gruppe, die sich aus den Datenschutzbeauftragten der EU-Mitgliedstaaten zusammensetzt, ist eindringlich gewarnt worden, dass die geplanten Maßnahmen einen starken Eingriff in das Leben praktisch jedes europäischen Bürgers darstellten und »eine riesige Fülle an Informationen über fast alle Kontakte, Interessen, Lebenswandel, Aufenthaltsorte und letztendlich auch das Tun, Denken und Fühlen« des Einzelnen verfügbar machen werden. ${ }^{25}$

Auch die Maßnahmen der EU zum Schutz der Außengrenzen (so die Erhöhung der Fälschungssicherheit von Reisedokumenten durch die Aufnahme biometrischer Daten oder die Erweiterung des Zugangs zu und die Vernetzung von VIS und EURODAC) illustrieren das Dilemma, in dem sich die EU befindet. Eine bedeutsame »Blindstelle« bei der Verhütung von Terroranschlägen besteht nämlich darin, dass der (illegale) Grenzübertritt, gegen den die EU und ihre Mitgliedstaaten umfangreiche Maßnahmen umgesetzt haben oder planen, in einer Vielzahl der Fälle gar nicht den ersten Schritt zur Vorbereitung eines Terroranschlages in Europa dargestellt hat. Islamistisch motivierte Terrorgruppen in Europa setzen sich aus unterschiedlichen salafistisch-jihadistischen ${ }^{26}$ Bewegungen zusammen, die ihren Ursprung in den arabisch-islamischen Staaten haben. Islamistische Terroristen haben sich in überwiegender Zahl jedoch nicht aus dem Kreis neu eingereister Drittstaatsangehöriger rekrutiert, sondern aus legal in Europa lebenden Einwanderern. ${ }^{27}$ Die an den Anschlägen von Madrid und London unmittelbar beteiligten Akteure hatten zuvor einige Jahre in der EU gelebt oder waren dort geboren worden. Sie waren in der EU überwiegend einer legalen Beschäftigung nachgegangen und hatten keine langjährige terroristische »Karriere« hinter sich gebracht.

Gegenwärtig ist eine Parallelentwicklung zu beobachten: Auf der einen Seite kooperieren islamistische Gruppen noch im-

24 Parlament und Rat der Europäischen Union, Richtlinie 2006/24/EG des Europäischen Parlaments und des Rates vom 15. März 2006 über die Vorratsspeicherung von Daten, die bei der Bereitstellung öffentlich zugänglicher elektronischer Kommunikationsdienste oder öffentlicher Kommunikationsnetze erzeugt oder verarbeitet werden, und zur Änderung der Richtlinie 2002/58/EG, Straßburg, 15. März 2006, PE-CONS 3677/12/05 REV 12.

25 Eighth Annual Report on the Situation Regarding the Protection of Individuals with Regard to the Processing of Personal Data in the European Union and in Third Countries, Brussels 2005, S. 5.

26 Der Salafismus versucht den Islam von allen äußeren Einflüssen zu reinigen. Jihadisten versuchen dieses Ziel auch mit gewaltsamen Mitteln zu erreichen.

27 Hierzu die bisher unveröffentliche Studie von Robert S. Leiken/Steven Brooke, A Quantitative Analysis of Terrorism and Immigration, The Nixon Center, Washington D.C., 25 January 2005. Siehe hierzu auch: Robert S. Leiken, Europe's Mujahideen. Where Mass Immigration Meets Global Terrorism, Center for Immigration Studies, April 2005, http://www.cis.org/ articles/2005/ back405.pdf; Robert S. Leiken/Steven Brooke, Al Qaeda's second front: Europe, in: International Herald Tribune vom 15. Juli 2005; Nikolas Busse, In den Dschihad mit europäischem Paß - Eine quantitative Studie zum islamistischen Terror in westlichen Ländern, in: Frankfurter Allgemeine Zeitung vom 22. Juli 2005. 
mer weltweit, auch scheint es in Europa eine »mittlere« Ebene zu geben, die religiöse Unterweisung erteilt, Rekrutierungen vornimmt, sowie finanzielle und logistische Hilfe leistet. AlQaida nahestehende Gruppen, die internationale Verbindungen aufweisen - wie al-Tawhid und Ansar El-Islam - haben seit 2001 wiederholt Anschläge in Europa vorbereitet. Einige Mitglieder dieser Gruppen hatten zwar eine paramilitärische Ausbildung in Camps der Al-Qaida oder der Taliban in Afghanistan erhalten. ${ }^{28}$ Von den eigentlichen »Führungspersonen « wie etwa Osama Bin Laden kann angenommen werden, dass sie (inzwischen) eher durch ihre religiöse Leitbildfunktion wirken, als durch die tatsächliche planerische Vorbereitung von Anschlägen oder direkte materielle Leistungen.

Während im Fall der Anschläge von Madrid die Frage von Hintermännern noch immer nicht eindeutig zu beantworten ist, ${ }^{29}$ ist im Falle von London erwiesen, dass die Attentäter hier offenbar nicht einmal mehr den lose kooperierenden islamistischen Netzwerken angehört haben; sie sind auch nicht von Hintermännern geführt worden. Die Gruppe hat sich Angaben des Haupttäters Mohammed Sidique Khan zufolge von Bin Laden und El-Zawahiri lediglich »inspirieren « lassen. ${ }^{30}$ Sie wollte den von ihnen als »ungerecht « empfundenen »Krieg « des Westens gegen den Islam auf die westlichen Metropolen ausweiten. ${ }^{31}$ Zwei Wochen später hat eine weitere Gruppe (erfolglos) versucht, die Anschläge von London nachzuahmen, ohne dass ein Zusammenhang zwischen den beiden Gruppen bestanden hat.

Nach den Erfahrungen insbesondere von London muss davon ausgegangen werden, dass islamistische Terroristen in Zukunft ihre Aktionen verstärkt autonom vorbereiten und nicht einmal mehr über internationale Netzwerke planen. Solche autonomen terroristischen Zellen bilden sich vor allem auf der Basis von Verwandtschaftsbeziehungen, Freundschaften, regionaler Herkunft oder dem gemeinsamen Einwandererstatus. ${ }^{32}$ Die Organisationsformen dieser Gruppen, das Täterprofil der Terroristen und die Verwendung leicht zu beschaffender oder herzustellender Sprengstoffe machen die Vorbereitung von Anschlägen nur schwer aufdeckbar. ${ }^{33}$

Langfristig aussichtsreicher zur Terrorismusprävention und aus dem Blickwinkel der Wahrung von Bürgerrechten allemal

28 Nesser, Jihad In Europe - A survey of the motivations for Sunni Islamist terrorism in post-millennium Europe, Forsvarets Forskningsinstitutt, Norwegian Defence Research Establishment, RAPPORT-2004/01146, S. 12, http:// www.mil.no/multimedia/archive/00039/Jihad_in_Europe_39602a.pdf.

29 Die Terroristen gehörten offenbar zu einer multinationalen Zelle, die (bisher nicht geklärte) Verbindungen zu radikalen Islamisten in anderen europäischen Staaten und in Nordafrika unterhalten hat.

30 Report of the Official Account of the Bombings in London on 7 th July 2005, London, S. 20-21, http://www.homeoffice.gov.uk/ documents/7-july-report.pdf.

31 Der Krieg im Irak hat zusätzlich vielerlei Gruppen in Europa aktiviert, die aber nur lose Verbindungen untereinander besitzen. Ihre Aktivitäten sind überwiegend auf die Vorbereitung von Anschlägen im Irak gerichtet. Vgl. Peter R. Neumann, Europe's Jihadist Dilemma, in: Survival , 48(2), 2006, S. 71-84 (77).

32 Vgl. Robert S. Leiken, Europe's Angry Muslims, in: Foreign Affairs 84 (2005):4, S. 120-135.; Robert S. Leiken/Steven Brooke, Al Qaeda's second front: Europe, International Herald Tribune, July 15, 2005.

33 Siehe hierzu Hartmut Behr, Terrorismusbekämpfung im Rahmen der Vereinten Nationen - Eine Bilanz der Anti-Terrorismuspolitik seit der SR Resolution 1373 (28.09.2001), in: Zeitschrift für Internationale Beziehungen 1/2004 S. 27-59 (31-32); Christopher Daase, Terrorismus: Der Wandel von einer reaktiven zu einer proaktiven Sicherheitspolitik der USA nach dem 11. September 2001, in: Christopher Daase/Susanne Feske/Ingo Peters (Hrsg.), Internationale Risikopolitik - Der Umgang mit neuen Gefahren in den internationalen Beziehungen, Baden-Baden 2002, S. 113-142 (117-118). unbedenklicher als viele der von der EU und ihren Mitgliedstaaten auf den Weg gebrachten Maßnahmen scheint die von Kommission und Rat erarbeitete Strategie zur Bekämpfung der Radikalisierung und Anwerbung für den Terrorismus. Durch sie soll verhindert werden, dass sich Menschen dem Terrorismus zuwenden und eine nächste Generation von Terroristen entsteht. Der Rat hat die möglichen Maßnahmen in diesem Bereich unter drei Hauptaspekten zusammengefasst: (1) Die Bekämpfung von Faktoren, die die Anwerbung erleichtern und von Aktivitäten solcher Netze und Personen, die Anwerbungsversuche für den Terrorismus vornehmen, (2) die Bekämpfung motivierender Faktoren, die zur Radikalisierung von Personen führen können, und (3) die Bekämpfung struktureller Faktoren, die das sozialökonomische Umfeld schaffen, in dem radikale Botschaften fruchtbar werden können. ${ }^{34}$ So sollen etwa Programme aufgelegt werden, die marginalisierte und schwer erreichbare Gruppen ansprechen und ihre Integration erleichtern.

Ganz unproblematisch ist jedoch auch diese Strategie nicht. Es bleibt erst noch abzuwarten, wie sich diese Maßnahmen und solche, die auf die Entwicklung eines Dialogs zwischen den Kulturen setzen, mit den ebenfalls in diesem Programm vorgesehenen polizeilichen Maßnahmen vertragen werden, bei denen Überwachung und strafbewehrte Beschränkungen von Handlungen im Vordergrund stehen. Der Rat stellt überdies zwar heraus, dass durch bestimmte gesellschaftliche Bedingungen ein Umfeld geschaffen werden kann, in dem Menschen sich leichter radikalisieren lassen. Aus seiner Sicht spielen die genannten Faktoren innerhalb der Gemeinschaft jedoch nur vereinzelt eine Rolle. Um der Radikalisierung und Anwerbung entgegenzuwirken, soll die EU nach Willen des Rates deshalb verstärkt außerhalb der Union zur Durchsetzung verantwortungsvoller Staatsführung, von Menschenrechten, Demokratie, Bildung und wirtschaftlicher Prosperität beitragen. ${ }^{35}$ Dass eine neue Tätergeneration innerhalb der Gesellschaften der EU herangereift ist und dies mit dort zu lokalisierenden sozialen Missständen zusammenhängen könnte, findet in der Strategie so zwar Berücksichtigung, letztendlich wird jedoch der Blick wieder auf von außen kommende Bedrohungen gerichtet.

\section{Fazit}

Die Prävention von terroristischen Anschlägen und die Verfolgung terroristischer Straftäter bleibt für die Union ein schwieriges Unterfangen. Mit der Behandlung des islamistischen Terrorismus nicht vorrangig als militärisches Problem, sondern als spezifische Form von Kriminalität, wird die EU der Problemstruktur des Terrorismus zwar im Kern gerecht, so wie sie seine Bekämpfung konkret angeht, bleibt allerdings eine Reihe von Zielkonflikten und Paradoxien. Zum einen lässt das Fehlen exekutiver Kompetenzen im Bereich der Terrorismusbekämpfung die grundsätzliche Frage aufkommen,

\footnotetext{
34 Rat der Europäischen Union, Strategie der Europäischen Union zur Bekämpfung von Radikalisierung und Anwerbung für den Terrorismus, Brüssel, 24. November 2005, 14781/1/05 REV 1, S. 3-5.
}

35 Rat der Europäischen Union, 14469/4/05 REV 4, a.a.O., S. 8-9. 
ob die EU langfristig überhaupt in der Lage ist, politische Führung in Bezug auf die Durchführung einer kohärenten Terrorismusbekämpfungspolitik in Europa zu übernehmen. Selbst die Koordinationsarbeit, die den größten Teil der Terrorismuspolitik der EU ausmacht, wird in der Hauptsache durch intergouvernementale Konsultationen bestimmt. ${ }^{36}$ Viele derjenigen Maßnahmen aber, die die EU bei der Terrorismusbekämpfung ergriffen hat oder plant, bedrohen zum anderen in erheblichem Maße Freiheits- und Bürgerrechte. Zudem liegt noch ein deutlicher Schwerpunkt auf der Identifizierung von Drittstaatenangehörigen, die illegal einreisen oder sich bereits mit gefälschten Papieren im Inneren der EU aufhalten. Diese Politik ist weder neu noch innovativ, noch stimmt eine solche Prioritätensetzung mit der Diagnose hinsichtlich der Autonomisierung des islamistischen Terrorismus überein. ${ }^{37}$

Terroristische Anschläge wie die von Madrid oder London können mit den beschriebenen Maßnahmen (vor allem zur verdachtsunabhängigen Überwachung im Binnenraum der EU und zum Schutz der Außengrenzen) kaum verhindert werden. Die beteiligten Akteure hatten in der EU gelebt oder waren dort geboren worden, waren durch einschlägige Straftaten nicht auffällig geworden und haben die Anschläge autonom vorbereitet. Ein Detail, das kaum Beachtung gefunden hat, dennoch von hoher Bedeutung für die Motive und das Vorgehen der islamistischen Terroristen ist, sollte jenen zu Denken geben, die Terrorismus durch Datenbanksysteme, Überwachungsmaßnahmen oder biometrische Identifizierungen bekämpfen wollen: Alle Attentäter von London haben bei den Anschlägen echte Ausweisdokumente mit sich geführt. ${ }^{38}$ Sie wollten ihre Identität gar nicht verschleiern, sondern sie offenbar vielmehr als Signal für Gleichgesinnte einsetzen, ihnen durch eigene Anschläge nachzufolgen. Wenn Terroristen aber ihre Identität wirklich verbergen wollten, könnten sie etwa die Überwachungsmaßnahmen im Telekommunikationsbereich, von denen die Bürgerinnen und Bürger in ihrem Alltagsleben direkt betroffen sind, durch die Nutzung von Telefonzellen, Internet-Cafés oder außerhalb der EU ansässigen Anbietern von E-Mail-Diensten oder Prepaid-Mobilfunkkarten relativ einfach umgehen. Zur Vereitelung von Anschlägen in Europa durch islamistische Terroristen haben bisher offenbar »klassische Mittel wie »verdachtsabhängige« Geheimdienstarbeit und Polizeiverhöre den größten Teil beigetragen. ${ }^{39}$

36 Doron Zimmermann, The European Union and Post-9/11 Counterterrorism: A Reappraisal, in: Studies in Conflict \& Terrorism, 29:123-145, 2006, S. 124

37 Siehe auch Thierry Balzacq/Sergio Carrera, The EU's Fight against International Terrorism Security Problems, Insecure Solutions, Centre for European Policy Studies, CEPS Policy Brief, No. 80/July 2005.

38 Report of the Official Account of the Bombings in London on 7th July 2005, London, S. 10-12, http://www.homeoffice.gov.uk/documents/7-july-report.pdf. 39 Nesser, Jihad In Europe, a.a.O., S. 29, 42, 52, 62.
Trotz der diskutierten Vorbehalte verspricht die EU-Strategie zur Bekämpfung der Radikalisierung und Anwerbung für den Terrorismus aus dem Blickwinkel der Prävention langfristig aussichtsreicher zu sein. Sie ist zumindest in Teilen innovativ und richtet sich - wenn auch noch allzu bruchstückhaft - auf die tieferliegenden Ursachen des Terrorismus. Ihre weitere Entwicklung und vor allem auch die in ihrem Rahmen zur Verfügung gestellten finanziellen Mittel werden zeigen, ob die EU in diesem wichtigen Bereich der Terrorismusbekämpfung eine langfristig angelegte Strategie entwickelt oder es bei einer wohlklingenden Rhetorik belässt. Die Anschläge von Madrid und London haben gezeigt, dass bislang nicht auffällig gewordene EU-Bürger zu islamistisch motivierten Terroristen geworden sind, eine Entwicklung, gegen die die gängigen Mittel der Kontrolle von Drittstaatsangehörigen, ja nicht einmal viele gängige Mittel der Kriminalitätsbekämpfung noch ausreichend greifen können. Umso mehr ist die EU gehalten, umfassende und abgestimmte Strategien zur langfristigen Bekämpfung des Nährbodens für den Terrorismus auch innerhalb der Union selbst zu entwickeln und zu implementieren.

Entscheidungen und Maßnahmen zur Bekämpfung des Terrorismus werden im Lichte einer Abwägung zwischen »Sicherheit « oder »Freiheit « auch in Zukunft schwierig und umstritten sein. ${ }^{40}$ Die Frage der Balance zwischen diesen beiden Polen und insbesondere die Frage, wie weit staatliche Gewalt bei der Bekämpfung des Terrorismus gehen darf, hat in den westlichen Demokratien bereits zu intensiven öffentlichen Kontroversen geführt. Beispielhaft zu nennen sind die mögliche Tolerierung von Folter (bzw. der Nutzung von unter Folter zustande gekommenen Vernehmungsergebnissen) und anderer extralegaler Praktiken durch EU-Mitgliedstaaten, ${ }^{41}$ wodurch grundsätzliche Fragen der Bewahrung und des Verlusts tradierter Werte und identitätsstiftender Normen in demokratischen Gesellschaften berührt sind. Die EU muss sich, nicht zuletzt aus Gründen der Legitimität und Akzeptanz bei den Bürgern deshalb intensiv mit der Frage auseinander setzen, wie, in welchem Ausmaß und zu welchen Kosten die Sicherheit durch solche Maßnahmen erhöht werden kann. Als Richtlinie muss stets die Frage dienen, ob die anvisierten Maßnahmen - insbesondere, wenn sie »invasiv « sind - angesichts der gegenwärtigen Entwicklungsrichtung des islamistischen Terrorismus überhaupt eine einigermaßen verlässliche Aussicht auf Wirkung haben.

40 Frank Gregory, The EU's Response to 9/11: A Case Study of Institutional Roles and Policy Processes with Special Reference to Issues of Accountability and Human Rights, in: Terrorism and Political Violence, 17:2005, S. 105-123.

41 Siehe hierzu Dick Marty (Rapporteur), Alleged secret detentions and unlawful inter-state transfers involving Council of Europe member states Draft report - Part II (Explanatory memorandum, Committee on Legal Affairs and Human Rights, AS/Jur (2006) 16 Part II, 7 June 2006, ajdoc 162006 Part II. 